Acid mantle 188

Acne 84

Adhesion molecule 405

Allergic contact dermatitis 59

Allergy 165

Amino acid adduct 59

Aminoglycosides 252

Androgen 356

Antiallergic drug 405

Antibacterial activity, Oriental herb extracts 84

Antigen 59

Antihistamines, topical 4

Antimicrobial agents 176

Antipruritic therapy 4

Apoptosis 50

Atopic dermatitis 367, 386, 405

Autoimmunity 117

Axon reflex 263

Bath PUVA therapy 305

Bax 50

Bcl-2 50

Bcl-x 50

Benzoquinone 59

Bexarotene 237

Bifidobacterium-fermented soy milk extract 108

Bioavailability 217, 305

Biometric techniques 367

Burns 313

Carrier protein 59

CD11b 405

Cell culture 156

- proliferation 379

Ceramides 291

Chemotaxis 84, 405

Cholesterol 291

Chromametry 151

Clinical score, skin aging 100

Coal tar 12

Collagen 242

Compound 48/80 165, 263
Contact allergy, occupational 117

Corticosteroid(s) 12,69

Cutaneous reaction 165

- T cell lymphoma 237

Cysteine 59

Cytokine 405

Dermal delivery 123

Dermatitis 259

Dermis 18

Differential scanning calorimetry 393

Dinitrofluorobenzene 165

Dithranol 12, 50, 69

DNA vaccine 271

Dutasteride 356

Eczema 367

Efficacy measurement 4

Electroporation 271

Enhancers, skin permeation 91

Eosinophils 405

Eotaxin 405

Epinastine 405

Erythema 237

17a-Estradiol 356

Fas 50

- ligand 50

Fibroblasts 143

Formulations, ibuprofen 5\% 137

Glucocorticoids 143

Glutathione 117

Glycosaminoglycan 242

Guidance, skin surface pH assessment 188

HaCaT keratinocytes 379

Hairless mice 108, 242

Hapten 59

Histamine 263

Horny layer profile 217
Human keratinocytes 108

- plasma 372

- skin 28, 343, 356

- - fibroblasts 108

- stratum corneum 393

Hyaluronic acid 108

Hydrocortisone 393

Ibuprofen 137

IgE 165

Interleukin-5 405

In vitro percutaneous permeation 91

- - predictions 123

- - tests 379

Ion pairs 156

Irritation 12

Isopropyl alcohol 393

- myristate 393

Isoprostanes 372

Itch 165

Keratinocytes 143

Keratolysis 151

Laser Doppler velocimetry 18

LGD-1069 237

Lichen planus 46

Lipid models 291

- peroxidation 36

Lipogenesis 84

Lipophilic ointment 91

Liposomal formulation 271

Lysine 59

Mass balance 343

- spectrometry 291, 372

Mast cells 263

Melatonin 379

8-Methoxypsoralen 305

Mexoryl SX ${ }^{\circledR} 343$

MicroAct 165

Microdialysis 18

Microemulsion 151, 271

\title{
KARGER
}

(C) 2003 S. Karger AG, Basel

Fax +4161306 1234

E-Mail karger@karger.ch Accessible online at:

www.karger.com www.karger.com/sph 
Minimal erythema dose 237

Moisturizer 367

Monohydroxyeicosatetraenoic acids 372

Mouse scratching behavior 165

$\mathrm{N}$-acetyltransferase 2386

Natural moisturizing factor 188

- preservatives 176

Nerves 46

Nitroflurbiprofen derivative 91

Normal human epidermal keratinocytes (NHEK) 252

\section{NSAIDs 137}

Optical spectroscopy 217,228

Organotypic culture 108

Oriental herbs 84

Oxidative stress 283, 291

Ozone 283

\section{Palmitoleic acid 176}

Passive cutaneous anaphylaxis 165

Percutaneous absorption 343

- -, clobetasol propionate 212

Permeability, tissue 130

Pharmacokinetic profile, 8-methoxypsoralen 305

Phenol 313

pH gradient 188

Photoaging 100

Photographic standard, skin aging 100

Photopheresis 372

Platelet-activating factor 259

Prednicarbate 143

Protein absorption 228
Pseudoabsorption 228

Psoralen 130

Psoriasis 12, 46, 69, 91, 130, 212, 237

- vulgaris 50

Quinone 59

Retinoids 46, 69

Rexinoid 237

Ribozymes 252

RNase P 252

RXR 237

Salicylic acid 69, 151

Scoring system skin aging 100

Sebum 176

Silver nitrate test 151

Skin 18, 100, 242, 263

- barrier 188

- ceramides 203

- diffusion activation energy 283

- distribution 130

- irritation 188

- lipids 176, 203

- microflora 188

- penetration 137

- permeability 203

- stripping 313

- surface pH 188

- - temperature 313

Slow acetylation 386

Specific protein staining 228

Squalene monohydroperoxide 242

Stratum corneum 28, 188, 203, $217,283,291$
Sun protection factor 28

Sunscreen 28, 343

Superoxide dismutase 36

Tape stripping 217, 228, 271

Tar 69

Targretin $^{\circledR} 237$

Tensides 156

Thalidomide 123

- analogues 123

Thermal injury 36

Topical corticosteroids 212

- formulations, ibuprofen 5\% 137

- gel 130

- vaccine 271

Toxicity, mixed tensides 156

Transactivation 143

Transdermal drug delivery 203

Transepidermal water loss 18,283

Ultraviolet B 237, 242

- filters 28

- light 259

- radiation 283,291

Vitamin $\mathrm{D}_{3}$ analogues 69

- E 36

Weighing 217

Wound maturation 313

Wrinkling 242

X-ray diffraction 393 